Methods A comprehensive literature search of published and unpublished studies (grey literature) in five electronic databases (MEDLINE, Embase, PsycINFO, the Cochrane Library and CINAHL) was conducted to identify randomized controlled trials (RCTs) that met the inclusion criteria. Supplementary searches in Google Scholar, hand searches in relevant journals, and backward and forward citation searching of included studies and relevant reviews were also conducted. A systematic approach to searching, screening, reviewing and data extraction was applied in accordance with the process recommended by the Cochrane Collaboration. Two researchers examined titles, abstracts, full-texts for eligibility independently. Risk of bias (RoB) was assessed using Cochrane RoB for randomized controlled trials. In addition, intervention and comparator group material were coded by two independent reviewers into their BCTs using the BCT taxonomy version 1. Disagreements were resolved by a consensus procedure.

Results The search of the predefined databases resulted in 6004 records, while a further 1334 records were found from other sources, giving a total of 7,338 citations. After duplicates were removed $(\mathrm{n}=3,241)$, a total of 4,097 citations were screened against the inclusion criteria. Of these, 4,012 citations were excluded on the basis of title, keywords, and abstract. The full texts of the remaining articles $(n=85)$ were then assessed against the inclusion criteria, resulting in 36 RCTs being retained. The studies were assessed as either low or unclear risk of bias. The majority of the RCTs investigated behaviour regarding antibiotic prescribing among physicians. The findings suggest that letters that incorporate social norms techniques are effective and can lead to behaviour change, with the potential for change in clinical outcomes. The effects might be enhanced by use of behavioural instruction, and a high-profile messenger.

Conclusion The evidence from this review provides important insights for healthcare providers and health authorities regarding how to formulate the communication, and what information and behaviour change techniques to include in order to optimise the potential effect on the behaviour of the receivers.

\section{P44 RISK-AWARENESS, ATTITUDE AND PRACTICE OF HEALTH CARE PROFESSIONALSTOWARDS REDUCING TOBACCO SMOKE EXPOSURE AMONG PREGNANT WOMEN AND CHILDREN}

\begin{abstract}
1,2ZZinab Hassanein*, 1,3 Ilze Bogdanovica, 1,3 Tessa Langley, 1,3 Rachael Murray,
1,3,4 Jo Leonardi-Bee. 'Division of Epidemiology and Public Health, University of Nottingham, Nottingham, UK; ${ }^{2}$ Public Health department, Assiut University, Assiut, Egypt; ${ }^{3}$ UK Centre for Tobacco and Alcohol Studies, University of Nottingham, Nottingham, UK; ${ }^{4}$ The Nottingham Centre for Evidence-Based Healthcare: JBI Institute, University of Nottingham, Nottingham, UK
\end{abstract}

\subsection{6/jech-2021-SSMabstracts. 132}

Background Approximately 50\% of Egyptian pregnant nonsmoking women and $55 \%$ of children are exposed to secondhand smoke (SHS), representing a major public health concern. Health care professionals (HCPs) are well placed to help in preventing exposure and its associated health harms by increasing risk-awareness in these groups. This study aimed to explore risk-awareness, attitudes and practice of HCPs in maternal and child health $(\mathrm{MCH})$ clinics in Egypt towards SHS exposure among pregnant women and children.

Methods A survey was distributed to all HCPs working in public $\mathrm{MCH}$ clinics in primary and secondary health care centres in Assiut city, Egypt in 2020. The questionnaire included 12 questions exploring risk-awareness, 10 questions for attitude and 5 questions for counselling practice of HCPs. Scores on risk-awareness were classified as 'low', and 'high' risk awareness, while attitudes towards SHS exposure were classified as 'low', and 'high' negative attitudes. Scores on counselling practices were classified as good practice if $75 \%$ of questions answered with always or sometimes. Univariate analysis was performed to explore factors associated with high risk-awareness, high negative attitude and good counselling practice.

Results 367 HCPs participated in the survey (response rate 68.5\%): 45\% nurses, 21\% gynaecologists/obstetricians, 16\% paediatricians, $9 \%$ general practitioners and $9 \%$ midwives. $12.5 \%$ of HCPs reported being smokers and $70.3 \%$ reported exposure to SHS in their workplace and $51.7 \%$ in their homes. $22.1 \%$ of participants reported having received training on smoking cessation. $55.9 \%$ had high awareness of the risks of SHS exposure to health of pregnant women and children. General practitioners and paediatricians had higher risk awareness than other specialities $(\mathrm{P}<0.001)$. 52.9\% of HCPs had high negative attitude towards SHS exposure among pregnant women or children. This finding was more common among HCPs who were female HCPs $(57.4 \%, \mathrm{P}=0.01)$, working in rural areas $(61.3 \%, \mathrm{P}=0.02)$ and who reported not being exposed to SHS at home $(59.9 \%, \mathrm{P}=0.001) .51 .8 \%$ of HCPs reported having good counselling practice regarding SHS exposure. This was significantly associated with being female $(56.2 \%, P=0.01)$, serving a rural population $(68.5 \%$, $\mathrm{P}=0.001)$, receiving previous training on smoking cessation (72.8\%, $\mathrm{P}=0.001)$ and not being exposed to SHS at home $(61.6 \%, \mathrm{P}=0.001)$.

Conclusion Egyptian HCPs' awareness, attitude and practice in relation to the risks of SHS to pregnant women and children is inadequate. It is essential for them to receive additional education and training to enable them to help pregnant women/mothers of children to avoid SHS exposure.

\section{P45 THE WE CAN QUIT2 SMOKING CESSATION TRIAL KNOWLEDGE EXCHANGE AND DISSEMINATION: PRIORITIES FROM A COMMUNITY PERSPECTIVE, AND RECOMMENDATIONS FOR FUTURE RESEARCH AND POLICY}

${ }^{1}$ Stefania Castello* ${ }^{1}$ Catherine Darker, ${ }^{2} J$ oanne Vance, ${ }^{3}$ Elaine Buckle ${ }^{3}$ Caitriona Reynolds, ${ }^{3}$ Aine Buggy, ${ }^{2}$ Kevin O'Hagan, ${ }^{4}$ Norma Cronin, ${ }^{5}$ Nadine Dougall, ${ }^{6}$ Declan Devane. ${ }^{1}$ Public Health and Primary Care, Trinity College Dublin, Dublin, Ireland; ${ }^{2}$ Community Cancer Prevention, Irish Cancer Society, Dublin, Ireland; ${ }^{3}$ Tobacco Free Ireland Programme, Health Service Executive, Dublin, Ireland; ${ }^{4}$ National Women's Council, Dublin, Ireland; ${ }^{5}$ School of Health and Social Care, Edinburgh Napier University, Edinburgh, UKi ${ }^{6}$ Trials Methodology Research Network and School of Nursing and Midwifery, HRB Trials Methodology Research Network and NUI Galway, Galway, Ireland; 'Usher Institute, University of Edinburgh, Edinburgh, UK

\subsection{6/jech-2021-SSMabstracts. 133}

Background We Can Quit (WCQ) is a tailored communitybased smoking cessation intervention comprising group support delivered by trained lay women with optional access to combination Nicotine Replacement Therapy (NRT) without charge. It was designed for women living in socioeconomically disadvantaged areas in Ireland. A pilot cluster randomised controlled trial (WCQ2) determined that WCQ was feasible and highly acceptable to the target group. A key aim of the trial 
Knowledge Exchange and Dissemination plan was to reengage with key stakeholders to present and request their feedback on trial findings, and to inform the design of a future definitive effectiveness trial.

Methods Stakeholders who supported recruitment of women and/or trial planning, representatives of the Irish Cancer Society, the Ireland's Health Service Executive, community organisations and pharmacies, GPs and primary care staff were invited to participate in an online interactive workshop. A Policy Brief which summarised trial findings was disseminated beforehand. Specific workshop objectives were to capture stakeholders' views on improvements to community engagement, strategies to enhance recruitment and retention, and policy and practice priorities arising from the research. Workshop participants were invited to fill in an anonymous, openended questionnaire after the workshop to register any further views on the previously discussed topics. Field notes taken during the workshop and questionnaire responses were combined to obtain a final list of challenges, barriers and recommendations for policy development and future research from a community perspective.

Results Forty-one stakeholders attended the workshop. The need for additional time to build relationships with local stakeholders for participant referral was identified. Further development of the social prescribing model in primary care was recommended as a vehicle to enhance recruitment. Low literacy was identified as a barrier to recruitment and retention, to be addressed by simplifying trial-related information, and by greater assistance with data completion. Other recommendations included provision of an intervention boost after trial completion to facilitate retention, and to maintain the established group support by encouraging participants to join other healthy community programmes. Key policy priorities were to remove cost and administrative barriers to access NRT, prioritise smoking cessation support tailored to disadvantaged groups, and to recognise and fund the peer-support model for smoking cessation.

Conclusion These results yielded important strategies to optimise the design of a future trial to assess WCQ effectiveness on smoking cessation for women smokers living in disadvantaged districts in Ireland. The findings may be generalisable to other community-based health interventions.

\section{P46 EVOLUTION OF GEOGRAPHICAL INEQUALITIES IN COVID-19 MORTALITY OVER THE FIRST WAVE OF THE PANDEMIC IN ENGLAND}

${ }^{1}$ Claire Welsh* ${ }^{*}$ 1,2 Viviana Albani, ${ }^{1,2}$ Fiona Matthews, ${ }^{1,2}$ Clare Bambra. ${ }^{1}$ Population Health Sciences Institute, Newcastle University, Newcastle, UK; ${ }^{2}$ Applied Research Collaboration North East and North Cumbria, Newcastle University, Newcastle, UK

\subsection{6/jech-2021-SSMabstracts. 134}

Background Early in the COVID-19 pandemic it was clear that inequalities were emerging in the distribution of deaths, with more deprived areas harder hit than less deprived ones. How these inequalities began, evolved, and changed with the implementation of the first national lockdown did not receive as much attention. We used COVID-19 death counts per local authority (LA) over the first wave (up to 4th July, 2020) to understand how COVID-19 impacted different areas, and to shed light on the equity effects of a strict national lockdown.
Methods Weekly COVID-19 death counts per LA were provided by the Office for National Statistics, along with population estimates for mid-2019 and data on the index of multiple deprivation (IMD) and median age per LA. Mortality rates for each area were calculated as deaths per 100,000 persons. Characteristics of the evolution of mortality rate per LA and decile of IMD were calculated, for example the speed of increase to each LAs peak rate. Simple linear models and descriptive statistics were used to compare areas.

Results More deprived LAs tended to begin recording COVID-19 deaths earlier than less deprived areas. Mortality rates in more deprived LAs rose at a faster rate to their peak, which was also higher than in less deprived LAs. All LA death rates peaked between 3 and 9 weeks following the implementation of the first lockdown. The time between the lockdown announcement and the peak death rate was significantly negatively associated with the speed of increase, adjusted for the median age of the population (95\% CI 0.33 to -0.31 deaths per 100,000 per week). Total cumulative mortality varied from 10.79 to 170.61 deaths per 100,000 persons per LA. Cumulative death rates were significantly higher in the most deprived $10 \%$ of LAs compared to others $(\mathrm{p}<0.001)$. Until lockdown, the most deprived $20 \%$ of LA's recorded $64 \%$ more deaths than the most deprived 20\%. By 4th July, this gap had narrowed to $16 \%$.

Conclusion Variation in the timing and speed of increase of local mortality rate curves occurred in the first wave and led to large inequalities across deprivation quintiles. This inequality was reduced but not completely abolished by the strict national lockdown imposed in March 2020. Timings of future national lockdowns should consider differences in local epidemic evolution, with extra support offered to those areas hardest hit.

\section{P47 INTERROGATING STRUCTURAL INEQUALITIES IN COVID- 19 MORTALITY IN ENGLAND AND WALES}

1,2 Gareth Griffith*, 1,2 George Davey Smith, ${ }^{4}$ David Manley, 1,2Laura Howe, ${ }^{3}$ Gwilym Owen. ${ }^{1}$ Medical Research Council Integrative Epidemiology Unit, University of Bristol, Bristol, UK; ${ }^{2}$ Population Health Sciences, Bristol Medical School, Bristol, UK; ${ }^{3}$ Department of Public Health and Policy, University of Liverpool, Liverpool, UK; ${ }^{4}$ School of Geographical Sciences, University of Bristol, Bristol, UK

\subsection{6/jech-2021-SSMabstracts. 135}

Background Numerous observational studies have highlighted structural inequalities in COVID-19 mortality in the UK. Such studies often fail to consider the complex spatial nature of such inequalities in their analysis, leading to the potential for bias and an inability to reach conclusions about the most appropriate structural levels for policy intervention.

Methods We use publicly available population data on COVID-19 related- and all-cause mortality between March and July 2020 in England and Wales to investigate the spatial scale of such inequalities. We propose a multiscale approach to simultaneously consider four spatial scales at which processes driving inequality may act and apportion inequality between these.

Results Adjusting for population age structure, number of care homes and residing in the North we find highest regional inequality in March and June/July. We find finer-grained within-region increased steadily from March until July. The 